\title{
Stroke and atrial septal defects - decision making at the turning of ages
}

\author{
Vlad Eugen Tiu', Athena Ribigan', Raluca Badea', Ovidiu Bajenaru1,2 \\ ${ }^{1}$ Department of Neurology, University Hospital Bucharest, Romania \\ ${ }^{2}$ Department of Clinical Neurosciences, \\ "Carol Davila" University of Medicine and Pharmacy, Bucharest, Romania
}

\begin{abstract}
CLOSE and Gore-REDUCE trials have recently changed the way we approach patients with stroke and patent foramen ovale. We present the case of a young patient with an Embolic Stroke of Undetermined Source (ESUS) and an atrial septal defect causing a large right-to-left shunt, with an ample discussion on evidence-based decision making.

Keywords: embolic stroke, atrial septal defect, cerebral artery
\end{abstract}

\section{INTRODUCTION}

In mid-May this year, the results of CLOSE and Gore-REDUCE trials were announced. It was the first time when large, randomized prospective trials proved the efficacy of patent foramen ovale (PFO) closure as compared to medical therapy alone. While guidelines are constantly adapting to this changes, decision making, when it comes to individuals, is still not an easy task.

\section{CASE PRESENTATION}

In June 2017, a 52 years old male working as a driver was admitted to our ward. He described waking up 2 days prior to admittance with an overall feeling of dizziness and visual disturbance, although he could not specify precisely what that was. He went to work and, after a couple of minutes in traffic, suffered a minor collision with a car coming from his right side. By the time he got to the hospital, all his symptoms were gone.

The usual culprits come to mind, but this was not the case. There was no family history of cerebrovascular disease, no cardiovascular risks, no smoking, he was fit and declared he had a rather athletic lifestyle.

On examination, both his neurological and general exam were all within normal range, but his description of the events strongly suggested transient right homonymous hemianopia.

Before admission, the patient had been referred by another physician to a cerebral MRI. Multiple ischemic lesions were present in both left and right internal carotid territories, but also in that of the right posterior cerebral artery, with 8 DWI restriction images in the right parieto-occipital territory, which correlated well with the recent symptoms (Fig. 1).

A cervical arteries Doppler Ultrasound was quickly performed, which showed a soft, ulcerated plaque on the right Internal Carotid Artery, resulting in a $40 \%$ occlusion of the vessel, without other significant atheromatous lesions.

Both arterial blood pressure and ECG rhythm were monitored for 48 and 24 hours, respectively, showing only moderate blood pressure elevation to $150 \mathrm{mmHg}$ in the latter part of the day, with no arrhythmic events recorded (Fig. 2). 

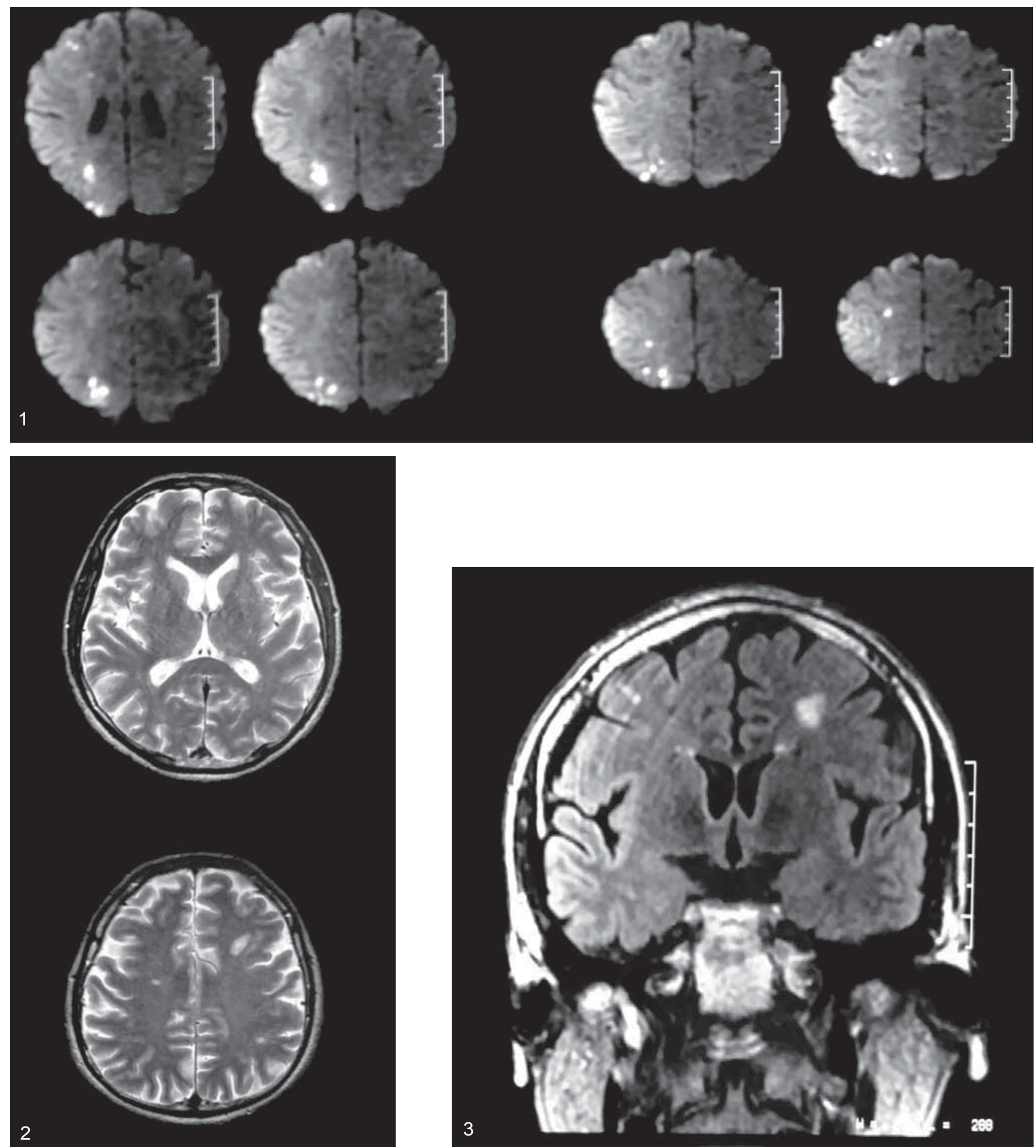

FIGURE 1. 1 - The DWI cerebral MRI shows multiple hyperintense signals in the right parieto-occipital lobes, suggesting recent ischemic injury; 2 - T2 axial sequence showing multiple hyperintense signals in both right and left carotid territories, with a large $(2 / 3 \mathrm{~cm})$ ischemic sequelae visible in the left frontal lobe;;3 - T2 FLAIR coronal sequence - periventricular, subcortical and deep white matter hyperintense signals, all signs of previous ischemic events, prove a worrying rate of recurrence for the 52 years old patient.

Bellow - US of the right internal carotid artery showing a soft, ulcerated plaque, resulting in $40 \%$ occlusion of the vessel.

The transthoracic echocardiography (TTE) found 2 small $(<3 \mathrm{~mm})$ atrial septal defects (ASD), with no other pathological findings (pulmonary hy- pertension, valvulopathies, etc.) Transesophageal echocardiography (TEE) proved positive for transient right-to-left-shunt (RLS) when contrast agent 


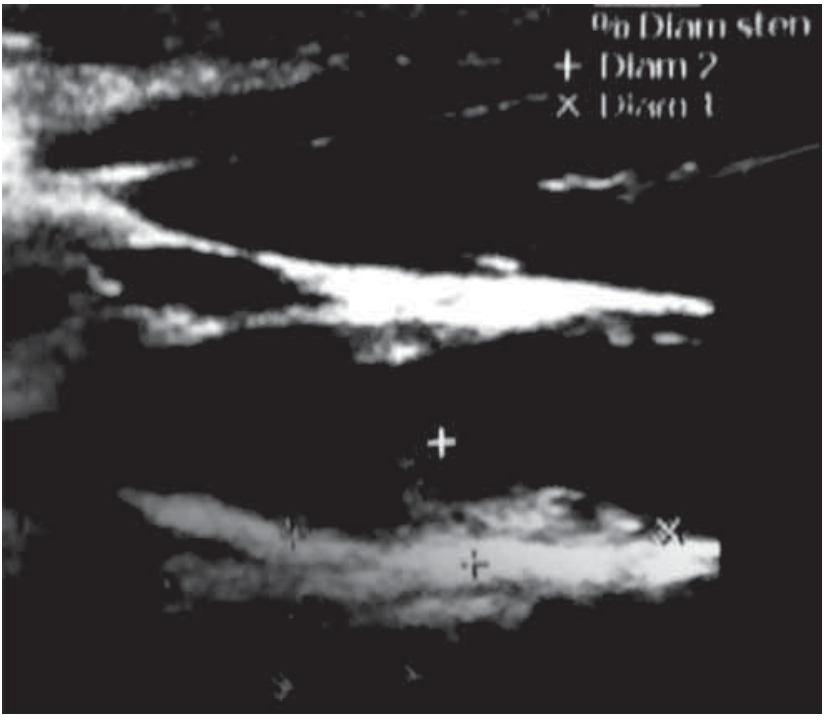

FIGURE 2

was injected. Pelvic and lower limbs Doppler US showed no signs of deep venous thrombosis.

The Angio-CT for supra-aortic arteries revealed that the patient had bilateral fetal posterior cerebral arteries, a common anatomical variant in which the posterior communicating artery is larger than the first segment (P1) of the posterior cerebral artery. This partially explained the distribution of the ischemic injuries on the cerebral MRI.

We then performed bilateral transcranial Doppler (b-TCD), with a 30 minutes microemboli detection protocol (negative), followed by contrast agent bolus injection (agitated saline-blood-air mix).This was highly positive with over 30 gaseous microemboli being detected on both middle cerebral arteries.

The patient was orally anticoagulated with Acenocumarol and referred to a cardiovascular surgery center for the closure of the ASD. Statin treatment was initiated for the atheromatous plaque on the RICA, and a blood work panel was recommended in order to investigate a potential hypercoagulable state.

\section{DISCUSSIONS}

There are at least three very interesting topics that arise from this case: whom should we refer to PFO or DSA closure? What is the most relevant mi-
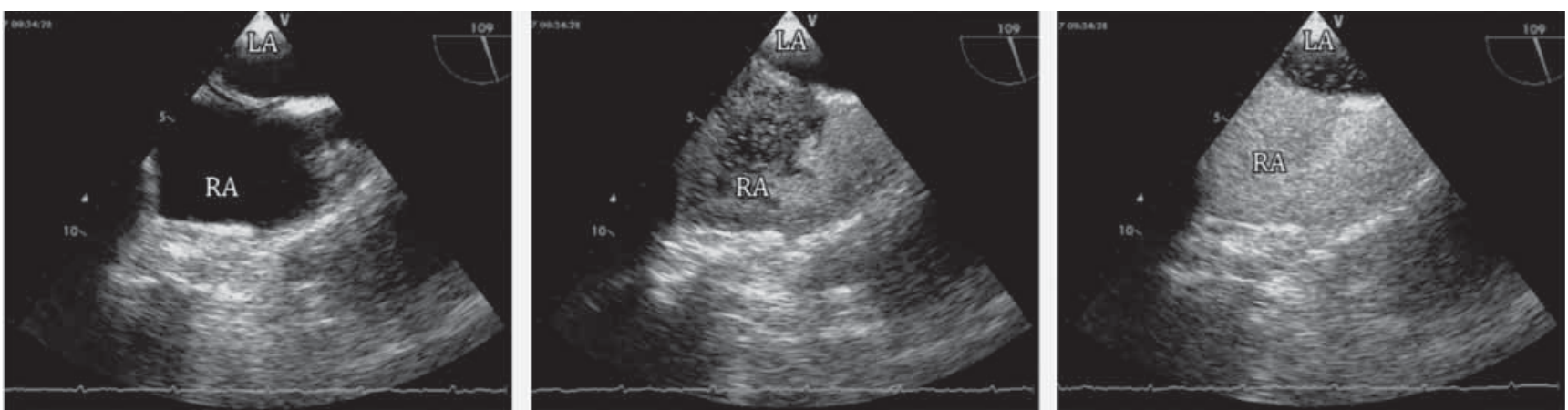

FIGURE 3. This series shows TEE contrast examination - the right atrium (RA) fills with contrast agent, then microbubbles can be easily seen passing through the ASD to the left atrium (LA) with each cardiac cycle. Well over 30 microbubbles were recorded in the LA during the examination.
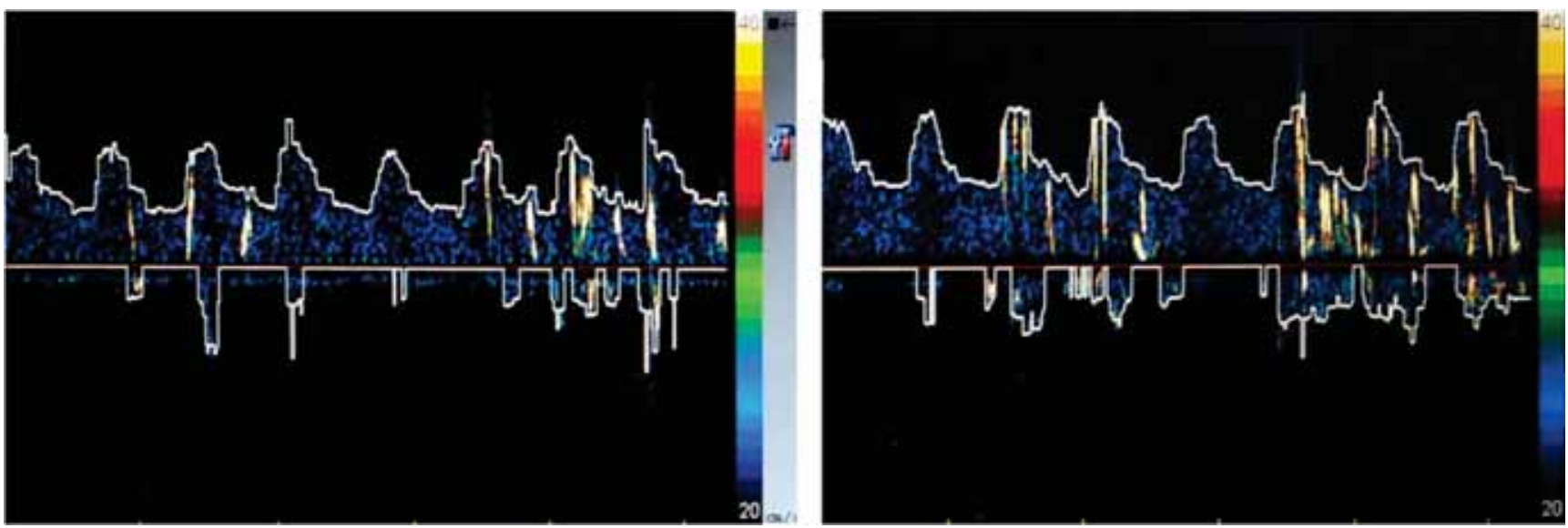

Figure 4. Contrast-Transcranial Doppler (c-TCD) with bilateral MCA monitoring - image shows gaseous microemboli detection (yellow spikes) on both the left and right MCA after agitated saline-blood IV injection. More than 30 microembolic signals (MES) were recorded during the examination. 
croemboli detection from a neurologist's point of view? What medical option should we recommend to our patients until they undergo a closing procedure or to those that have no indications for surgical therapy?

The issue of whom benefits from patent foramen ovale (or other forms of ASD) closure has been long standing. If we are to examine the recent developments on the matter, it was in 2008 that the American Association of Cardiology released a new Guideline regarding the management of adults with congenital heart disease. The authors considered that patients should be referred to interventional options once clear right ventricle (RV) overload was proven or pulmonary hypertension was diagnosed. In regards to our current case, the closure of an ASD was considered reasonable in the presence of a paradoxical embolism (Class IIa, level of evidence C). Small ASDs with a diameter of less than $5 \mathrm{~mm}$ and no evidence of RV volume overload were considered to be rather harmless unless associated with a paradoxical embolism. (1)

Proving a paradoxical embolism, however, is not an easy task. For most patients presenting with a cryptogenic stroke and a PFO or other ASD, a source of embolization will not be found (2). Numerous mechanisms are thought to be at play: a) the paradoxical embolic event, a passage of a thrombus from the peripheral venous system through the atrial septum defect; b) the thrombus is generated inside the atriums in relationship with a PFO-generated arrhythmia (atrial fibrillation being the main culprit); c) formation of the thrombus in the septum defect and lastly, d) PFO related hypercoagulability (3). While this are all largely accepted theories, supporting evidence for the last two is still scarce.

Furthermore, it would be natural to assume that a patient that shows no signs of right ventricle volume overload or pulmonary hypertension would present only a left-to-right shunt, as in, from high to low pressure. It was proven, however, that transient instantaneous right-to-left shunt does occur even for these patients in certain conditions, such as early ventricular systole, when right atrial (RA) pressure briefly exceeds left atrial (LA) pressure (RA systole finishes after LA systole), or following a Valsalva maneuver (4).

A new concept of cryptogenic stroke has been coined in the last couple of years - the Embolic
Stroke of Undetermined Sources (ESUS). This is defined as a non-lacunar brain infarct without proximal arterial stenosis ( $<50 \%$ cervical arteries stenosis), cardioembolic sources or other specific causes of stroke identified (arteritis, dissecton, migraine, drug missuse, etc.). The work-up is extensive and requires that a brain CT/MRI, an ECG and 24h Holter monitoring be performed, TTE and either cervical duplex and TCD US or Angio-CT or Angio-MRI of the cervical and cerebral arteries examinations (5). Our patient met this criteria and can therefore be diagnosed with an ESUS.

Some may argue that, even though the RICA plaque resulted in only a $40 \%$ stenosis, its soft, ulcerated aspect and the greater number of ischemic events on the right carotid territory may bear some significance. While non-stenotic carotid artery plaques are considered to carry a low absolute risk of stroke, small studies have shown that ulcerated, irregular ones may be a source of arteriogenic thromboembolism. A small study of 32 patients with cryptogenic stroke and non-stentoic carotid arteries plaque showed that $38 \%$ had type VI complicated plaques on MRI on the ipsilateral artery versus $0 \%$ on the contralateral one (6). Considering that our patient had bilateral ischemic lesions, no criteria for atherothrombotic stroke and that all vascular events had occurred under no medical therapy, the RICA plaque probably bared little clinical importance, and initiation of statin and antithrombotic therapies were the right choice until future check-ups.

ASD are not rare occurrences. Up to $10-35 \%$ of the general population has a PFO (7), so even in the setting of an embolic stroke of unknown sources, is it warranted to assume that the ASD and the ischemic event are correlated?

Many studies and analyses were performed in the recent years trying to find an answer to this question, and perhaps one of the most elegant solutions was brought to us by David M. Kent et al., who devised an index to identify stroke-related versus incidental findings of a patent foramen ovale in cryptogenic stroke. Their analysis led to the creation of a score based on six easily obtainable data - history of hypertension, diabetes, stroke or TIA, smoking, the presence of cortical infarcts on cerebral imaging and patient's age. This score was called RoPE (Risk of Paradoxical Embolism), and 
the lower it is, the lower the probability of causality (for example, for a score of 0-3 the prevalence of PFO for this group was $23 \%$ and the PFO-attributable fraction of strokes was $0 \%$ ). For patients with higher scores, the prevalence was shown to be much higher and the PFO-attributable fraction grew significantly (for a score of 9-10 points, the prevalence of $\mathrm{PFO}$ was $73 \%$ and the fraction was $88 \%)(8)$.

TABLE 1. The RoPE score calculator (8)

\begin{tabular}{|l|c|}
\hline Characteristic & Points \\
\hline No history hypertension & 1 \\
\hline No history of diabetes & 1 \\
\hline No history of stroke or TIA & 1 \\
\hline Nonsmoker & 1 \\
\hline Cor cal infarct on imaging & 1 \\
\hline \multicolumn{2}{|c|}{ Age (in years) } \\
\hline $18-29$ & 5 \\
\hline $30-39$ & 4 \\
\hline $40-49$ & 3 \\
\hline $50-59$ & 2 \\
\hline $60-69$ & 1 \\
\hline$\geq 70$ & 0 \\
\hline
\end{tabular}

Let's pause for a moment and use this score to evaluate our patient. With 7 points out of 10, the prevalence of PFO for this group was $54 \%$ and the likelihood that the cryptogenic stroke was in relation to the ASD was $72 \%$.

So what next?

First, medical treatment should be instated. Second, the patient should be referred to a cardiovascular surgeon for a pre-op evaluation.

There are many types of ASD. Sinus venosus, coronary sinus, and ostium primum defects are closed surgically, as they are not usually approachable by percutaneous device closure. Secundum ASD and PFO are usually solved by percutaneous intervention unless there are other associated conditions, whereas an ASA can be solved either way depending on its morphology (9).

If we are to compare percutaneous closure to open surgery, recent data seems to suggest that the percutaneous option is safer. A large study comparing 1270 patients in the surgical group to 1812 patients in the percutaneous group proved that the early complications rate, major complications rate and length of hospital stay were all greater for the surgical group, whereas the success rate was simil$\operatorname{lar}(10)$.
But is there really a benefit to closing the ASD rather than opting for medical therapy alone? For a long time, the answer was "No".

The first large randomized trial to address this question was CLOSURE. A total of 909 patients were randomly assigned to closure with percutaneous device or medical therapy alone, but there were no significant differences between the two groups. ${ }^{11}$

The PC trial enrolled 414 patients, of whom 204 were randomly assigned to the closure group and 210 to the medical-therapy group, but again finding no significant reduction in the risk of recurrent embolic events or death in the closure group, as compared with the medical-therapy one (12).

The largest of these studies was RESPECT trial, in which 980 patients were enrolled; 499 were randomly assigned to the closure group and 481 to the medical-therapy group. Once again, there was no significant benefit of closure of a patent foramen ovale over medical therapy alone in the intention-to-treat analysis (13).

The inclusion criteria for all these trials were similar - age between 18 to 60 years, a positive contrast bubble study by TEE, demonstrating right to left shunting through PFO (either spontaneous or during Valsalva), and a history of stroke or TIA in the last 6-9 months.

So why did recent trials succeed where three others had failed?

You'll notice that while both PC, RESPECT and CLOSURE trials had inclusion criteria asking for a positive contrast TEE for right-to-left shunt, none further refined this important aspect in order to select patients that were likely to benefit from the closure.

The inclusion criteria for the CLOSE trial were very similar with a key difference - the presence of a PFO with at least one of the characteristics: large shunt ( $>30$ microbubbles on TEE or TTE) or PFO associated with ASA at TEE showing a base of the aneurysm $>15 \mathrm{~mm}$ and excursion $>10 \mathrm{~mm}$.

Of the 663 patients included in CLOSE, 238 were allocated to PFO closure, 238 to antiplatelet therapy and 187 to oral anticoagulants. In the comparison between PFO closure and antiplatelet therapy, there was a significant reduction in rate of stroke recurrence for the closure group, but also increase in atrial fibrillation $(4,6 \%$ vs. $0,9 \%$, $\mathrm{P}<0.001)$. There was no significant difference be- 
tween the anticoagulation group versus antiplatelet therapy, even though a positive trend was seen for the anticoagulation group (14).

Gore-REDUCE trial was quite similar (the main difference was the type of occluder being used), with 664 patients being randomly assigned in a 2:1 ratio to either PFO closure or antiplatelet therapy alone. There was a significant reduction in recurrence rate for the closure group, but this study showed, too, a rise in atrial fibrillation for this group (6,6\% versus $0.4 \%)$ (15).

A key point, therefore, is not only demonstrating the microembolic events but also being able to quantify it. While TEE has become largely available, it is still a costly, time consuming, semi-invasive maneuver that has risks on its own. Serious complications may be rare $(0,2 \%)(16)$, but if we add in intolerance to the probe and all other events that lead to an unsuccessful examination, numbers are as high as 1 to $3 \%(17,18)$. A much safer and cheaper approach could be contrast-TCD, with bilateral monitoring of the MCA. A study performed in 2009 examined 107 patients with PFO, assessing RLS by simultaneous c-TCD and c-TEE. While the results were not impressive for small and medium shunts, when 2 or more microembolic signals were recorded on c-TCD, they predicted a large PFO on c-TEE (defined as 30 or more microbubbles), with a sensitivity, specificity and accuracy of 96.3, 96.8, and $96.9 \%$, respectively (19).

We'd like to stress out that, more than TEE, c-TCD gives a unique view to the vascular territory that a Neurologist is mostly interested in - the brain. Coming back to our case, if 2 or more MES on either of the MCA strongly correlates with a large shunt through the PFO, then what conclusions can be drawn from 30 MES on both MCA?

At this moment, the answer is that we don't know. New studies are needed to investigate, correlate and validate this investigation, and until then, no medical decision can be based upon it.

Our patient was scheduled for percutaneous closure of the ASD a month away from his discharge from our ward. So should he be released on antiplatelet therapy or oral anticoagulation? And if oral anticoagulation is chosen, then which one?

No study has this far proven the superiority of anticoagulation over antiplatelet therapy in preventing the recurrence of cryptogenic stroke, whether it was associated with a PFO or not.
In WARSS trial, for example, 2206 patients with stroke (with or without PFO) were randomized to aspirin (325 mg/day) or warfarin (target, INR 1.42.8 ), with rates of stroke recurrence, death or hemorrhage at 2 year follow-up being similar (20). Another study from Laussane prospectively followed 140 patients with PFO and cryptogenic stroke, although the treatment of either aspirin $(250 \mathrm{mg} /$ day), warfarin (objective, INR=3.5) or surgical closure was left at the physician's choice. No statistically significant difference was observed between the groups (21).

Perhaps of most interest is a sub-analysis of WARSS trial, the PICSS study. The main result was assessing that, on medical therapy, the presence of $\mathrm{PFO}$ in stroke patients did not increase the chance of adverse events regardless of PFO size or the presence of atrial septal aneurysm. However, when the groups were analyzed as a comparison between aspirin or warfarin medical therapy, no significant differences were found for the time to primary events (rate of stroke, TIA, or death at 2 years) (22). A more recent meta-analysis reached a similar conclusion (23).

So, case closed? Not quite. While some authors consider the recurrence of stroke for these patients to be so small under Aspirin that the added risk of anticoagulant therapy is not justified, others argue that therapy should be adjusted to the individual and not forget that, even if a clear source of venous thrombosis is not found, some patients should be treated as for such (24).

Our patient had a very low risk for bleeding, while showing a great number of recurrent ischemic events on the cerebral MRI. But what tipped the scale towards oral anticoagulation was the nature of his job: he was a driver, often staying in driver's seat for 8 to 10 hours at a time. Even if pelvic and lower limbs venous Doppler US could not prove any DVT, we still believed this to be the culprit and initiated anticoagulation.

In theory, anticoagulation and ESUS (related to an ASD or not) should work like a charm. As previously stated, the two main suspects in ESUS are either atrial fibrillation or deep vein thrombosis, both of which are currently a clear indication for oral anticoagulation. Even more so, we know now from the CRYSTAL AF and EMBRACE trials that the more you look for atrial fibrillation in crypto- 
genic stroke patients, the more you'll find it (12.4\% for continuous monitoring group vs. $2 \%$ for regular follow-up at 12 months in CRYSTAL $\mathrm{AF}^{25}$ and $16.1 \%$ of patients who received with 30 -day event recorder, versus only $3.2 \%$ by 24 -hour monitoring in EMBRACE) (26). Whatever the reason (poorly designed studies, overestimating recurrence rates and the possible outcomes, theoretical models that often oversimplify reality), warfarin has failed time and again to prove superior to antiplatelet therapy (27).

Currently restricted to prevention of stroke due to atrial fibrillation, prophylaxis and treatment of deep venous thromboembolism (28), NOACS may gain new indications in the years to come, as three large studies currently under way are trying to show their superiority to aspirin in prevention of ESUS recurrence. RE-SPECT ESUS is planning on randomizing 6,000 patients with cryptogenic stroke on either Dabigatran or Aspirin (29), NAVIGATE ESUS is randomizing 7,000 patients on Rivaroxaban or Aspirin (30), and ATTICUS is planning on enrolling 500 patients to prove the superiority of

Can a NOAC be prescribed to a young patient suffering an ESUS with a strong correlation to an ASD at this stage? Anticoagulation altogether is a decision that, lacking other indications, is outside Apixaban to Aspirin (31).

the guidelines. While Warfarin (and vitamin $\mathrm{K}$ antagonists) has been proven by clinical trials to be equal, if not better, than aspirin in prevention of stroke recurrence, no data exists on NOACs. If a high suspicion of DVT or atrial fibrillation persists even after a thorough work-up, one could argue that the NOAC is prescribed for the underlying cause and not the ESUS. It would have to be a shared decision, and a highly individualized one to say the least.

Once the results will be in, perhaps the guidelines will be rewritten and NOACs will enter their predicted golden age. But as far as ASD and stroke go, it is unlikely that decision making will ever be a walk in the park.

\section{CONCLUSION}

Current evidence supports either antiplatelet therapy for ASD related ESUS or, in case of large right-to-left-shunts, percutaneous or surgical closure. Anticoagulation should be reserved to few, selected patients in whom the benefits greatly outweigh the risk or those who have associated conditions requiring this treatment. NOACs are currently being investigated as an alternative in three large studies.

Conflict of interest: none declared Financial support: none declared

\section{REFERENCES}

1. Warnes C.A. et al. ACC/AHA 2008 Guidelines for the Management of Adults with Congenital Heart Disease: Executive Summary: a report of the American College of Cardiology/American Heart Association Task Force on Practice Guidelines (writing committee to develop guidelines for. Circulation 118, 2395-2451 (2008).

2. Wilbur J. \& Shian B. Diagnosis of deep venous thrombosis and pulmonary embolism. Am. Fam. Physician 86, 913-919 (2012).

3. Cruz-Gonzalez I., Solis J., Inglessis-Azuaje I. \& Palacios I. F. [Patent foramen ovale: current state of the art]. Rev. Esp. Cardiol. 61, 738-751 (2008)

4. Gomperts N., Fowler R., Horlick E. \& McLaughlin P. A broken heart: Right-to-left shunt in the setting of normal cardiac pressures. The Canadian Journal of Cardiology 24, 227-229 (2008).

5. Hart R.G. et al. Embolic strokes of undetermined source: the case for a new clinical construct. Lancet. Neurol. 13, 429-438 (2014).

6. Freilinger T.M. et al. Prevalence of nonstenosing, complicated atherosclerotic plaques in cryptogenic stroke. JACC. Cardiovasc. Imaging 5, 397-405 (2012).

7. Fisher D.C., Fisher E.A., Budd J.H., Rosen S.E. \& Goldman M.E. The incidence of patent foramen ovale in 1,000 consecutive patients. A contrast transesophageal echocardiography study. Chest 107 , 1504-1509 (1995).

8. Kent D.M. et al. An index to identify stroke-related vs incidental patent foramen ovale in cryptogenic stroke. Neurology 81, 619-625 (2013).
9. Heidi M. Connolly, MD, F. Surgical and percutaneous closure of atrial septal defects in adults. Uptodate 1-20 (2017).

10. Butera G. et al. Percutaneous versus surgical closure of secundum atrial septal defects: a systematic review and meta-analysis of currently available clinical evidence. EuroIntervention J. Eur. Collab. with Work. Gr. Interv. Cardiol. Eur. Soc. Cardiol. 7, 377-385 (2011).

11. Furlan A.J. et al. Closure or Medical Therapy for Cryptogenic Stroke with Patent Foramen Ovale. N. Engl. J. Med. 366, 991-999 (2012).

12. Meier B. et al. Percutaneous Closure of Patent Foramen Ovale in Cryptogenic Embolism. N. Engl. J. Med. 368, 1083-1091 (2013).

13. Carroll J. D. et al. Closure of Patent Foramen Ovale versus Medical Therapy after Cryptogenic Stroke. N. Engl. J. Med. 368, 1092-1100 (2013).

14. Mas J.-L. et al. Closure of patent foramen ovale, oral anticoagulants or antiplatelet therapy to prevent stroke recurrence: Study design. Int. J. Stroke 11, 724-732 (2016).

15. Sue Hughes. Finally, Success Reducing Recurrent Stroke With PFO Closure. Medscape (2017). Available at: http://www.medscape.com/ viewarticle/880102. (Accessed: 16th July 2017)

16. Daniel W.G. et al. Safety of transesophageal echocardiography. A multicenter survey of 10,419 examinations. Circulation $83,817-821$ (1991).

17. Mathur S.K. \& Singh P. Transoesophageal Echocardiography Related Complications. Indian Journal of Anaesthesia 53, 567-574 (2009). 
18. Gendreau M.A., Triner W.R. \& Bartfield J. Complications of transesophageal echocardiography in the ED. Am. J. Emerg. Med. 17, 248-251 (1999)

19. Kobayashi K. et al. Contrast transcranial Doppler can diagnose large patent foramen ovale. Cerebrovasc. Dis. 27, 230-234 (2009).

20. Hankey G.J. Warfarin-Aspirin Recurrent Stroke Study (WARSS) Trial. Stroke 33, 1723 LP-1726 (2002).

21. Bogousslavsky J., Garazi S., Jeanrenaud X., Aebischer N. \& Van Melle G. Stroke recurrence in patients with patent foramen ovale: the Lausanne Study. Lausanne Stroke with Paradoxal Embolism Study Group. Neurology 46, 1301-1305 (1996).

22. Homma S., Sacco R.L., Di Tullio M.R., Sciacca R.R. \& Mohr J.P. Effect of Medical Treatment in Stroke Patients With Patent Foramen Ovale. Circulation 105, 2625 LP-2631 (2002).

23. Kitsios G.D., Dahabreh I.J., Abu Dabrh A.M., Thaler D.E. \& Kent D.M. Patent foramen ovale closure and medical treatments for secondary stroke prevention: a systematic review of observational and randomized evidence. Stroke 43, 422-431 (2012).

24. Wessler B.S. Prevention of Recurrent Stroke in Patients with Patent Foramen Ovale. 33, 491-500 (2015).

25. Sanna T. et al. Cryptogenic Stroke and Underlying Atrial Fibrillation. N. Engl. J. Med. 370, 2478-2486 (2014).
26. Gladstone D.J. et al. Atrial Fibrillation in Patients with Cryptogenic Stroke. N. Engl. J. Med. 370, 2467-2477 (2014).

27. Toth P.P. Considerations for long-term anticoagulant therapy in patients with venous thromboembolism in the novel oral anticoagulant era. Vascular Health and Risk Management 12, 23-34 (2016).

28. Clinical Excellence Commission. Non-vitamin K Antagonist Oral Anticoagulant (NOAC) Guidelines. (2016).

29. 29. Diener, H.-C. et al. Design of Randomized, double-blind, Evaluation in secondary Stroke Prevention comparing the EfficaCy and safety of the oral Thrombin inhibitor dabigatran etexilate vs. acetylsalicylic acid in patients with Embolic Stroke of Undetermined Source (RE-SPECT E. Int. J. Stroke 10, 1309-1312 (2015).

30. 30. Bayer. Rivaroxaban Versus Aspirin in Secondary Prevention of Stroke and Prevention of Systemic Embolism in Patients With Recent Embolic Stroke of Undetermined Source (ESUS) (NAVIGATE ESUS). (2014). Available at: https://clinicaltrials.gov/ct2/show/NCT02313909.

31. 31. University Hospital Tuebingen. Apixaban for Treatment of Embolic Stroke of Undetermined Source (ATTICUS). December 2015 Available at: https://clinicaltrials.gov/ct2/show/NCT02427126. (Accessed: 19th July 2017) 